\title{
Medo odontológico e cárie dentária em crianças: uma revisão crítica da literatura
}

\author{
Isla Camilla Carvalho Laureano, Lunna Farias, Liege Helena Freitas Fernandes, \\ Alessandro Leite Cavalcanti* \\ Departamento de Odontologia, Faculdade de Odontologia, Universidade Estadual da Paraíba, \\ Campina Grande, PB, Brasil
}

Histórico do Artigo:
Recebido em:
13/10/2020
Aceito em:
29/10/2020

Palavras-chave: Ansiedade ao tratamento odontológico; cárie dentária; criança

Keywords:

Dental anxiety; dental caries; child

\begin{abstract}
RESUMO
O medo odontológico é um problema grave que afeta negativamente a saúde bucal. Esta revisão crítica da literatura objetivou identificar os estudos existentes sobre o medo odontológico associado à experiência de cárie dentária em crianças. Tratou-se de estudo bibliográfico realizado nas bases de dados PubMed, Web of Science e Scopus, por meio dos artigos indexados até outubro de 2019. Os termos utilizados foram: "Dental fear", "Dental anxiety", "Dental caries" e "Children". As informações coletadas foram: autor, o ano de publicação, país, desenho do estudo, número amostral, faixa etária (em anos), o instrumento utilizado para avaliar o medo odontológico e os principais resultados. Os dados foram analisados utilizando-se o Microsoft Excel, sendo apresentados descritivamente. Foram obtidos 533 artigos e 28 foram incluídos. A maioria dos estudos foram realizados por pesquisadores brasileiros $(32,1 \%)$. O número de participantes variou de 99 a 2734 crianças. A idade mais frequente foi de 12 anos $(59,3 \%)$ e o instrumento mais utilizado foi o questionário Children's Fear Survey Schedule-Dental Subscale (CFSS-DS) $(32,1 \%)$, com os respondentes sendo as próprias crianças $(82,1 \%)$. A prevalência de medo odontológico variou de $5,2 \%$ a $66,0 \%$, e os valores de escore médio variaram de 1,50 a 37,0 . Os estudos sobre o medo odontológico associado à experiência de cárie dentária em crianças foram realizados em maior número no Brasil, utilizando o questionário CFSS-DS. Existe ampla variabilidade na prevalência e valores de escore médio em diferentes países.
\end{abstract}

Dental fear and dental caries in children: a critical review of literature

Dental fear is a serious problem that negatively affects children's oral health. This critical review of the literature aimed to identify existing studies on dental fear associated with the experience of dental caries in children. This bibliographic study was carried out in PubMed, Web of Science, and Scopus databases through articles indexed until October 2019. The terms used were: "Dental fear", "Dental anxiety", "Dental caries" and "Children". The information collected were: author, year of publication, country, design of study, sample number, age group (in years), questionnaire used to assess dental fear, and the main results. The data were analyzed using Microsoft Excel, being presented descriptively. A total of 533 articles were obtained, and 28 were included. Most studies were carried out by Brazilian researchers $(32.1 \%)$. The number of participants ranged from 99 to 2,734 children. The most frequent age was 12 years old (59.3\%), and the most used questionnaire was the Children's Fear Survey Schedule-Dental Subscale (CFSS-DS) (32.1\%), with the respondents being the children themselves $(82.1 \%)$. The prevalence of dental fear ranged from $5.2 \%$ to $66.0 \%$, and the mean score values ranged from 1.50 to 37.0. Studies on dental fear associated with the experience of dental caries in children were carried out in greater numbers in Brazil, using the CFSS-DS questionnaire. There is wide variability in prevalence and mean score values in different countries.
\end{abstract}

\section{Introdução}

O medo odontológico é um problema generalizado que acomete indivíduos de todas as idades, podendo levar à recusa do tratamento e impactar negativamente as saúdes bucal e psicológica dos indivíduos (1). Ele se refere a uma reação emocional natural e desagradável a estímulos ameaçadores específicos que ocorrem em situações associadas ao tratamento odontológico (2).

\footnotetext{
*Autor correspondente: alessandrouepb@gmail.com (Cavalcanti A.L.)
} 
A prevalência do medo odontológico na infância varia significativamente na literatura, sendo que tal variabilidade pode ser explicada por aspectos relativos à diversidade das populações estudadas, considerando diferenças nos aspectos culturais $(3,4)$, nas faixas etárias avaliadas (5), nos instrumentos de pesquisa utilizados $(4,5)$, nas questões metodológicas, nos métodos de amostragem (5) e nos pontos de corte (3).

Embora o medo odontológico tenha natureza complexa e multidimensional, sua origem está associada a fatores relacionados aos aspectos comportamentais e da personalidade dos pacientes, à experiência de dor e de trauma durante o tratamento odontológico, ao perfil sociodemográfico e à observação ou imitação de modelos $(3,4,6)$. O impacto que essa condição pode ter na vida das pessoas é amplo e dinâmico, e o temor ao tratamento odontológico gera um problema cíclico, pois indivíduos com medo odontológico são mais propensos a postergar o tratamento, levando a problemas dentários mais severos e a padrões de visitas sintomáticas, os quais ampliam a exacerbação do medo existente (7).

Embora a Odontologia enfatize a necessidade de consultas preventivas, a cárie dentária é uma das principais razões para a primeira visita da criança ao cirurgiãodentista (8). A relação existente entre o medo odontológico e a ocorrência de cárie dentária é controversa (9). Crianças com maior índice de cárie e com menor nível de medo odontológico possivelmente compreendem que o profissional pode aliviar a sua dor, por isso não temem o tratamento odontológico (10), enquanto, aquelas crianças com maior índice de cárie e com maior medo odontológico, são mais propensas a relatar dor dentária (11), o que pode aumentar o medo (12).

Face à importância do tema e a necessidade de prover uma melhor compreensão dos fatores envolvidos, o presente estudo objetivou identificar, por meio de uma revisão crítica da literatura, os estudos existentes sobre o medo odontológico associado à experiência de cárie dentária em crianças. Espera-se evidenciar a necessidade de estratégias preventivas eficazes para melhorar a condição de saúde bucal de crianças e de intervenções para reduzir o medo odontológico.

\section{Método}

Tratou-se de estudo bibliográfico, cuja pergunta norteadora configurou-se em: "O medo odontológico está associado à experiência de cárie dentária em crianças?"

A busca foi realizada através das bases PubMed (US National Library of Medicine National Institutes of Health), Web of Science (Clarivate Analytics) e Scopus (Elsevier), por meio dos artigos indexados até outubro de 2019. Os termos utilizados foram: "Dental fear", "Dental anxiety", "Dental caries" e "Children", extraídos do Medical Subject Headings (MESH), e os operadores booleanos "AND" e "OR

Os critérios de inclusão adotados foram: estudos observacionais (transversal, coorte e caso-controle) e experimentais (ensaios clínicos randomizados e não randomizados); nos idiomas português ou inglês; com data de publicação entre janeiro de 2009 a outubro de 2019; os quais apresentavam associação entre o medo odontológico da criança e a cárie dentária; utilizavam o índice CPO-D/ceo-d, CPO-S/ceo-s ou International Caries Detection \& Assessment System (ICDAS), para avaliar a experiência de cárie; incluíam crianças sem transtornos neuropsiquiátricos, síndromes ou defeitos de desenvolvimento do esmalte; usavam escalas ou questionários validados direcionados ao medo odontológico das crianças e demonstravam valores do escore médio, mediana ou prevalência do medo odontológico.

A seleção dos artigos foi realizada através de uma leitura prévia dos títulos e resumos das referências encontradas. A análise dos resumos foi realizada por um único examinador. Os artigos selecionados foram acessados na íntegra, sendo realizada sua 
leitura e avaliação. As informações coletadas foram: autores, ano de publicação, país, tipo de estudo, número amostral, faixa etária (em anos) dos participantes, instrumento utilizado para avaliar o medo odontológico e principais resultados.

Os dados foram tabulados e analisados utilizando-se o Microsoft Excel 2016 para Windows (Microsoft Press, Redmond, WA, USA) e apresentados por meio de estatística descritiva (distribuições absolutas e percentual).

\section{Resultados}

Inicialmente, foram encontrados 533 artigos, sendo 213 no PubMed, 63 no Web of Science e 257 no Scopus. Depois da remoção dos artigos duplicados, permaneceram 293 publicações, das quais 37 foram escolhidas a partir da leitura do título e resumo. Desses estudos, nove foram excluídos por não atenderem aos critérios de elegibilidade e 28 foram selecionados para análise na íntegra. Após a leitura, os 28 artigos foram incluídos nesta revisão (Figura 1).

Em relação à distribuição geográfica, a maior parte dos estudos foram realizados por pesquisadores do Brasil (32,1\%), seguidos pela Índia (21,4\%). Quanto ao tipo de estudo, a maioria utilizou o desenho transversal $(75,0 \%)$. O número de participantes variou de 99 crianças (13) a 2734 crianças (14). A idade mais frequente foi de 12 anos $(59,3 \%)$ e 9 anos (51,8\%), variando de 2 anos (15) a 16 anos (16) (Quadro 1).

Os instrumentos mais utilizados para avaliar o medo odontológico foram o Children's Fear Survey Schedule-Dental Subscale (CFSS-DS) (32,1\%) e a Dental Anxiety Question (DAQ) $(21,4 \%)$ e os respondentes predominantes foram as próprias crianças $(82,1 \%)$. A prevalência de medo odontológico variou de 5,2\% de crianças espanholas (17) e turcas (18), com elevado nível de medo odontológico, utilizando o Index of Dental Fear and Anxiety (IDAF-4C+) e o CFSS-DS, respetivamente, a 66,0\% entre crianças brasileiras, utilizando a Facial Image Scale (FIS) (19). Os valores de escore médio variaram de 1,50, utilizando o IDAF-4C+ (20) a 37,0, empregando o CFSS-DS (21) (Quadro 1).

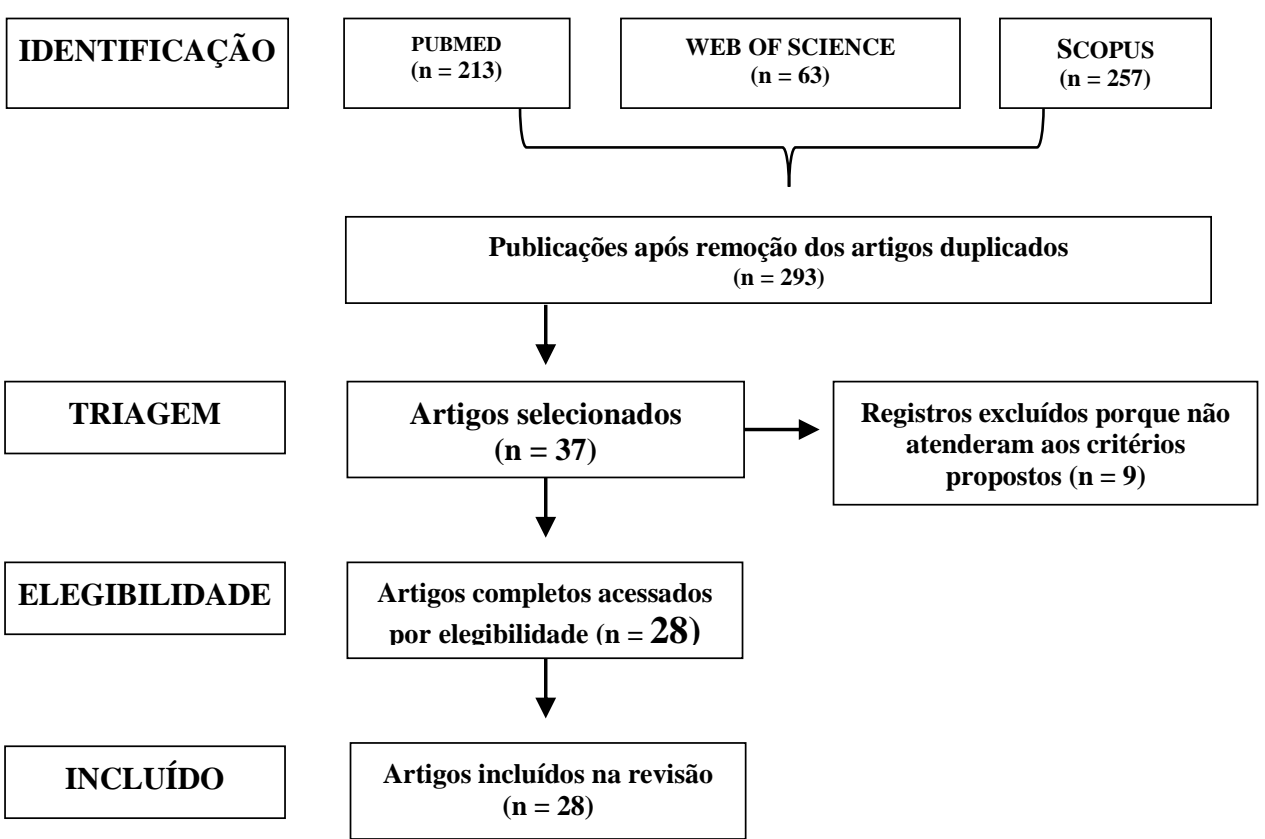

Figura 1 - Fluxograma do procedimento de seleção para os artigos incluídos na revisão. 
Quadro 1 - Artigos sobre o medo odontológico associado à experiência de cárie dentária.

\begin{tabular}{|c|c|c|c|c|c|c|c|}
\hline Autor & Ano & País & $\begin{array}{c}\text { Desenho } \\
\text { do } \\
\text { Estudo }\end{array}$ & Amostra & $\begin{array}{l}\text { Faixa } \\
\text { Etária } \\
\text { (anos) }\end{array}$ & Instrumento & Resultados \\
\hline $\begin{array}{l}\text { Akbay Oba et } \\
\text { al. (22) }\end{array}$ & 2009 & Turquia & Transversal & 275 & 7 a 11 & $\begin{array}{l}\text { CFSS-DS } \\
\text { Respondente: } \\
\text { Criança }\end{array}$ & $\begin{array}{l}\text { Prevalência: } 14,6 \% \\
\text { A diferença entre o nível de medo } \\
\text { odontológico e a experiência de cárie } \\
\text { dentária foi estatisticamente } \\
\text { significativa }(\mathrm{p}<0,05) \text {. Verificou-se } \\
\text { correlação entre medo odontológico e } \\
\text { cárie dentária }(\mathrm{p}<0,01) \text {. }\end{array}$ \\
\hline $\begin{array}{l}\text { Nicolas et al. } \\
\text { (23) }\end{array}$ & 2010 & França & Transversal & 1.303 & 5 a 12 & $\begin{array}{c}\text { Dental Fear - } \\
\text { Visual Analogue } \\
\text { Scale (DF-VAS) } \\
\text { Respondente: } \\
\text { Criança }\end{array}$ & $\begin{array}{l}\text { Prevalência: } 24,3 \% \\
\text { As crianças com, ao menos, um dente } \\
\text { cariado extensamente apresentaram } \\
\text { nível de medo odontológico maior } \\
\text { que aquelas sem cárie ( } \mathrm{p}=0,01 \text { ), } \\
\text { enquanto as crianças com dentes } \\
\text { restaurados foram significativamente } \\
\text { menos ansiosas que aquelas sem } \\
\text { tratamento odontológico anterior } \\
(\mathrm{p}<0,05) \text {. }\end{array}$ \\
\hline $\begin{array}{l}\text { Pieper et al. } \\
\text { (24) }\end{array}$ & 2012 & Alemanha & $\begin{array}{l}\text { Ensaio } \\
\text { Clínico }\end{array}$ & 420 & 12 & $\begin{array}{l}\text { Dental Fear in } \\
\text { Children } \\
\text { Questionnaire } \\
\text { Respondente: } \\
\text { Criança }\end{array}$ & $\begin{array}{l}\text { Escore médio: Grupo Teste: } 2,51 \pm \\
3,63 \text {; Grupo Controle: } 3,35 \pm 3,87 \\
\text { As crianças com maior experiência } \\
\text { de cárie dentária tiveram maiores } \\
\text { valores de medo odontológico, em } \\
\text { ambos os grupos. }\end{array}$ \\
\hline $\begin{array}{c}\text { Barbosa et al. } \\
\text { (25) }\end{array}$ & 2012 & Brasil & Transversal & 145 & 8 a 14 & \begin{tabular}{|c|} 
Revised Children's \\
Manifest Anxiety \\
Scale (RCMAS) \\
Respondente: \\
Criança
\end{tabular} & $\begin{array}{l}\text { Escore médio: } 14,9 \pm 6,9 \text { para } \\
\text { crianças de } 8 \text { a } 10 \text { anos; } 16,3 \pm 6,3 \\
\text { para pré-adolescentes de } 11 \text { a } 14 \text { anos } \\
\begin{array}{l}\text { Não foram observadas associações } \\
\text { significativas entre medo } \\
\text { odontológico e experiência de cárie } \\
\text { dentária. }\end{array}\end{array}$ \\
\hline $\begin{array}{l}\text { Olak et al. } \\
\text { (26) }\end{array}$ & 2013 & Estônia & Transversal & 344 & 8 a 10 & $\begin{array}{c}\text { Modified CFSS-DS } \\
\text { Respondente: } \\
\text { Criança }\end{array}$ & $\begin{array}{l}\text { Prevalência: } 6,1 \% \\
\text { As crianças cujos escores ceo-d / } \\
\text { CPO-D foram }>0 \text { tiveram escores de } \\
\text { medo odontológico mais elevados } \\
\text { que aquelas cujos escores ceo-d / } \\
\text { CPO-D foram }=0(\mathrm{p}<0,01) \text {. }\end{array}$ \\
\hline Beena (21) & 2013 & Índia & Transversal & 444 & 6 a 12 & $\begin{array}{l}\text { CFSS-DS } \\
\text { Respondente: } \\
\text { Criança }\end{array}$ & $\begin{array}{l}\text { Escore médio: } 37,0 \pm 8,89 \\
\text { Foram observadas diferenças } \\
\text { significativas entre as crianças de } 6 \\
\text { anos com CFSS-DS }<38 \text { e CFSS-DS } \\
\geq 38 \text { nos valores médios de ceo-s } \\
(p<0,05) \text { e entre as crianças de } 9 \text { anos } \\
\text { nos valores médios de ceo-d ( }<<0,01) \\
\text { e ceo-s ( }<<0,01) \text {. } \\
\text { Foram observadas diferenças } \\
\text { significativas entre as crianças de } 7 \\
\text { anos com CFSS-DS }<38 \text { e CFSS-DS } \\
\geq 38 \text { nos valores médios de CPO-D e } \\
\text { CPO-S (p }<0,05) \text { entre as crianças de } \\
10 \text { anos nos valores médios de CPO- } \\
\text { D e CPO-S (p<0,01) e entre as } \\
\text { crianças de } 12 \text { anos nos valores de } \\
\text { CPO-S (p<0,05). }\end{array}$ \\
\hline
\end{tabular}


Vittalle - Revista de Ciências da Saúde v. 32, n. 3 (2020) 266-277

\begin{tabular}{|c|c|c|c|c|c|c|c|}
\hline Autor & Ano & País & $\begin{array}{c}\text { Desenho do } \\
\text { Estudo }\end{array}$ & Amostra & $\begin{array}{l}\text { Faixa } \\
\text { Etária } \\
\text { (anos) }\end{array}$ & Instrumento & Resultados \\
\hline $\begin{array}{l}\text { Murthy et al. } \\
\text { (27) }\end{array}$ & 2014 & Índia & Transversal & 1.452 & 12 a 15 & $\begin{array}{c}\text { DAQ } \\
\text { Respondente: } \\
\text { Criança }\end{array}$ & $\begin{array}{l}\text { Prevalência: } 25,6 \% \text { com elevado } \\
\text { medo odontológico. } \\
\text { A prevalência e a mediana de cárie } \\
\text { dentária não foram associadas ao } \\
\text { medo odontológico } \quad(\mathrm{p}=0,23 \\
\mathrm{p}=0,18) \text {. }\end{array}$ \\
\hline $\begin{array}{l}\text { Torriani et al. } \\
\text { (28) }\end{array}$ & 2014 & Brasil & Coorte & 1.129 & 5 & $\begin{array}{c}\text { DAQ } \\
\text { Respondente: Mães }\end{array}$ & $\begin{array}{l}\text { Prevalência: } 16,8 \% \\
\text { A associação entre medo } \\
\text { odontológico e cárie dentária } \\
\text { demonstrou que crianças com ceo-s } \geq \\
2 \text { foram mais propensas a ter medo } \\
\text { odontológico que aquelas que } \\
\text { estavam livres de cárie dentária } \\
(\mathrm{p}<0,001) \text {. }\end{array}$ \\
\hline $\begin{array}{l}\text { Pichot et al. } \\
\text { (14) }\end{array}$ & 2014 & $\begin{array}{c}\text { Nova } \\
\text { Caledônia }\end{array}$ & Transversal & 2.734 & 6,9 e 12 & $\begin{array}{c}\text { DF-VAS } \\
\text { Respondente: } \\
\text { Criança }\end{array}$ & $\begin{array}{l}\text { Prevalência: Mais de } 15 \% \text { das } \\
\text { crianças de } 9 \text { a } 12 \text { anos tiveram } \\
\text { escores elevados de medo } \\
\text { odontológico (DF-VAS >6) } \\
\text { Aos } 12 \text { anos, as crianças com níveis } \\
\text { mais baixos de medo odontológico } \\
\text { (DF-VAS <3) tiveram menos } \\
\text { experiência de cárie dentária que as } \\
\text { crianças com níveis mais elevados } \\
(\text { DF-VAS }>6 \text { ). }\end{array}$ \\
\hline $\begin{array}{l}\text { Viswanath e } \\
\text { Krishna (16) }\end{array}$ & 2015 & Índia & Transversal & 529 & 12 a 16 & \begin{tabular}{|} 
Modified Corah's \\
Dental Anxiety \\
Scale \\
Respondente: \\
Criança
\end{tabular} & $\begin{array}{l}\text { Escore médio: } 9,91 \pm 3,40 \\
\text { O aumento do medo odontológico } \\
\text { levou a um aumento na incidência de } \\
\text { cárie dentária. }\end{array}$ \\
\hline $\begin{array}{l}\text { Carrillo-Diaz } \\
\text { et al. (17) }\end{array}$ & 2015 & Espanha & Transversal & 250 & 12 a 14 & $\begin{array}{l}\text { IDAF-4C+ } \\
\text { Respondente: } \\
\text { Criança }\end{array}$ & $\begin{array}{l}\begin{array}{l}\text { Prevalência: } 5,2 \% \text { apresentavam } \\
\text { medo odontológico acentuado } \\
\text { (escore IDAF } \geq 3 \text { ) }\end{array} \\
\text { Ter mais dentes cariados apresentou } \\
\text { correlação com o nível mais elevado } \\
\text { de medo odontológico, embora } \\
\text { tenha-se apresentado baixa. }\end{array}$ \\
\hline $\begin{array}{c}\text { Kakkar et al. } \\
\text { (1) }\end{array}$ & 2016 & Índia & Transversal & 250 & 10 a 14 & $\begin{array}{l}\text { CFSS-DS } \\
\text { Respondente: } \\
\text { Criança }\end{array}$ & $\begin{array}{l}\text { Prevalência: } 42,0 \% \\
\text { Crianças com maiores valores de } \\
\text { CPO-D apresentaram aumento dos } \\
\text { escores do CFSS-DS. }\end{array}$ \\
\hline $\begin{array}{l}\text { Bhalla et al. } \\
\text { (20) }\end{array}$ & 2017 & Índia & Transversal & 200 & $\begin{array}{c}\text { Idade } \\
\text { média } \\
13,5\end{array}$ & $\begin{array}{l}\text { IDAF-4C+ } \\
\text { Respondente: } \\
\text { Criança }\end{array}$ & $\begin{array}{l}\text { Escore médio: Grupo de atendimento } \\
\text { regular } 1,60 \text { e Grupo de atendimento } \\
\text { irregular 1,50. } \\
\text { Correlação significativa fo } \\
\text { observada entre maior número de } \\
\text { dentes cariados e aumento do nível } \\
\text { de medo odontológico. }\end{array}$ \\
\hline
\end{tabular}




\begin{tabular}{|c|c|c|c|c|c|c|c|}
\hline Autor & Ano & País & $\begin{array}{c}\text { Desenho do } \\
\text { Estudo }\end{array}$ & Amostra & $\begin{array}{l}\text { Faixa } \\
\text { Etária } \\
\text { (anos) }\end{array}$ & Instrumento & Resultados \\
\hline $\begin{array}{l}\text { Barreto et al. } \\
\text { (29) }\end{array}$ & 2017 & Brasil & Transversal & 1.367 & 6 e 7 & $\begin{array}{c}\text { DAQ } \\
\text { Respondente: } \\
\text { Criança }\end{array}$ & $\begin{array}{l}\text { Prevalência: } 54,4 \% \\
\text { Não foram observadas associações } \\
\text { significativas entre ansiedade } \\
\text { odontológica e experiência de cárie } \\
\text { dentária, porém houve associação } \\
\text { entre ansiedade odontológica e } \\
\text { dentes cariados }(\mathrm{p}<0,001) \text {. }\end{array}$ \\
\hline $\begin{array}{l}\text { Abanto et al. } \\
\text { (19) }\end{array}$ & 2017 & Brasil & $\begin{array}{l}\text { Ensaio } \\
\text { Clínico }\end{array}$ & 100 & 3 a 5 & $\begin{array}{c}\text { FIS } \\
\text { Respondente: } \\
\text { Criança }\end{array}$ & $\begin{array}{l}\text { Prevalência: } 66,0 \% \\
\text { Nem o nível de experiência de cárie } \\
\text { nem a severidade da cárie dentária } \\
\text { foram associados à ansiedade } \\
\text { odontológica. }\end{array}$ \\
\hline $\begin{array}{l}\text { Vilas-Boas et } \\
\text { al. (30) }\end{array}$ & 2017 & Brasil & Transversal & 100 & 6 a 11 & $\begin{array}{c}\text { Venham Picture } \\
\text { Test (VPT) } \\
\text { Respondente: } \\
\text { Criança }\end{array}$ & $\begin{array}{l}\text { Prevalência: } 24,0 \% \\
\text { Houve associação significativa entre } \\
\text { nível de ansiedade e cárie dentária } \\
(\mathrm{p}<0,001) \text {. }\end{array}$ \\
\hline $\begin{array}{l}\text { Soares et al. } \\
\text { (31) }\end{array}$ & 2017 & Brasil & Coorte & 416 & 5 a 7 & $\begin{array}{c}\text { DAQ } \\
\text { Respondente: Pais }\end{array}$ & $\begin{array}{l}\text { Prevalência: No início do estudo, } \\
\text { elevada ansiedade odontológica de } \\
16,2 \% \text {; enquanto, no } \\
\text { acompanhamento, foi de } 19,8 \% \text {. } \\
\text { Um aumento de uma unidade no } \\
\text { escore do ceo-d aumentou o risco de } \\
\text { ansiedade odontológica elevada em } \\
1,1 \text { vezes no acompanhamento. }\end{array}$ \\
\hline Boka et al. (8) & 2017 & Grécia & Transversal & 1.484 & 6 a 12 & $\begin{array}{l}\text { CFSS-DS } \\
\text { Respondente: } \\
\text { Criança }\end{array}$ & $\begin{array}{l}\text { Escore médio: } 27,1 \pm 10,8 \text {. } \\
\text { A experiência de cárie dentária não } \\
\text { se correlacionou com o medo } \\
\text { odontológico. Foi observada } \\
\text { correlação negativa entre o medo e o } \\
\text { número de dentes decíduos } \\
\text { restaurados. }\end{array}$ \\
\hline $\begin{array}{l}\text { Costa et al. } \\
\text { (15) }\end{array}$ & 2017 & Brasil & Coorte & 540 & 2 e 3 & $\begin{array}{c}\text { DAQ } \\
\text { Respondente: Mãe }\end{array}$ & $\begin{array}{l}\text { Prevalência: } 21,6 \% \\
\text { Crianças com medo odontológico } \\
\text { não demonstraram associação com a } \\
\text { experiência de cárie }(p=0,747) \text {. }\end{array}$ \\
\hline $\begin{array}{l}\text { Silveira et al. } \\
\text { (12) }\end{array}$ & 2017 & Brasil & Transversal & 1.202 & 8 a 12 & $\begin{array}{c}\text { DAQ } \\
\text { Respondente: } \\
\text { Criança }\end{array}$ & $\begin{array}{l}\text { Prevalência: } 24,6 \% \\
\text { Crianças que apresentavam dentes } \\
\text { cariados [RP }=1,32 \text { (IC } 95 \%: 1,01- \\
1,72 \text { )] mostraram-se mais propensas } \\
\text { a ter medo odontológico. }\end{array}$ \\
\hline $\begin{array}{l}\text { Balasubraman } \\
\text { ian et al. (32) }\end{array}$ & 2018 & Índia & Transversal & 227 & 3 a 6 & $\begin{array}{c}\text { FIS } \\
\text { Respondente: } \\
\text { Criança }\end{array}$ & $\begin{array}{l}\text { Prevalência: } 38,3 \% \text { apresentaram } \\
\text { elevada ansiedade. } \\
\text { Crianças com elevada ansiedade } \\
\text { odontológica apresentaram maior } \\
\text { média de dentes perdidos. }\end{array}$ \\
\hline
\end{tabular}


Vittalle - Revista de Ciências da Saúde v. 32, n. 3 (2020) 266-277

\begin{tabular}{|c|c|c|c|c|c|c|c|}
\hline Autor & Ano & País & $\begin{array}{c}\text { Desenho do } \\
\text { Estudo }\end{array}$ & Amostra & $\begin{array}{l}\text { Faixa } \\
\text { Etária } \\
\text { (anos) }\end{array}$ & Instrumento & Resultados \\
\hline $\begin{array}{l}\text { Alsadat et al. } \\
\text { (9) }\end{array}$ & 2018 & $\begin{array}{l}\text { Arábia } \\
\text { Saudita }\end{array}$ & Transversal & 1.546 & 6 a 12 & $\begin{array}{l}\text { CFSS-DS } \\
\text { Respondente: } \\
\text { Criança }\end{array}$ & $\begin{array}{l}\text { Prevalência: } 23,5 \% \text { das } \\
\text { apresentaram elevado } \begin{array}{r}\text { medodo } \\
\text { odontológico }(\text { CFSS-DS } \geq 32) ; 12,5 \%\end{array} \\
\text { tinham medo odontológico grave } \\
\text { (CFSS-DS> 38) } \\
\text { A severidade da cárie dentária } \\
\text { apresentou diferença significativa em } \\
\text { crianças que tiveram maiores escores } \\
\text { de medo odontológico }(\mathrm{p}=0,035) \text {. }\end{array}$ \\
\hline $\begin{array}{l}\text { Alshoraim et } \\
\text { al. (33) }\end{array}$ & 2018 & $\begin{array}{l}\text { Arábia } \\
\text { Saudita }\end{array}$ & Transversal & 1.522 & 12 a 15 & $\begin{array}{l}\text { CFSS-DS } \\
\text { Respondente: } \\
\text { Criança }\end{array}$ & $\begin{array}{l}\text { Escore médio: } 25,99 \pm 9,3 \\
\text { O nível de cárie dentária não foi } \\
\text { associado ao medo odontológico. }\end{array}$ \\
\hline $\begin{array}{l}\text { Folayan et al. } \\
\text { (34) }\end{array}$ & 2018 & Nigéria & Transversal & 450 & 6 a 12 & $\begin{array}{c}\text { CFSS-DS } \\
\text { Respondente: Mãe } \\
\text { (6 a } 7 \text { anos) e } \\
\text { Criança ( } 8 \text { a } 12 \\
\text { anos) }\end{array}$ & $\begin{array}{l}\text { Prevalência: } 52,7 \% \text { de ansiedade } \\
\text { odontológica elevada (CFSS-DS } \\
\geq 35 \text { ) } \\
\text { Crianças que apresentavam elevada } \\
\text { ansiedade odontológica exibiram } \\
\text { maiores chances de ter cárie dentária, } \\
\text { quando comparadas às crianças com } \\
\text { baixa ansiedade odontológica. }\end{array}$ \\
\hline $\begin{array}{c}\text { Buldur e } \\
\text { Armfield (35) }\end{array}$ & 2018 & Turquia & Transversal & 421 & 12 a 14 & \begin{tabular}{|c} 
CFSS-DS e IDAF- \\
4C+ \\
Respondente: \\
Criança
\end{tabular} & $\begin{array}{l}\text { Escore médio do IDAF-4C+: } 3,15 \pm \\
1,09 \\
\text { Ambas as medidas de medo } \\
\text { odontológico, o IDAF-4C e CFSS- } \\
\text { DS, foram significativamente } \\
\text { associados a cárie dentária }(\mathrm{p}<0,001) \text {. }\end{array}$ \\
\hline $\begin{array}{l}\text { Yahyaoglu et } \\
\text { al. (18) }\end{array}$ & 2018 & Turquia & Transversal & 810 & 6 a 12 & $\begin{array}{l}\text { CFSS-DS } \\
\text { Respondente: } \\
\text { Criança }\end{array}$ & $\begin{array}{l}\text { Prevalência: } 5,2 \% \text { de elevado nível } \\
\text { de medo odontológico (CFSS-DS } \\
\geq 39 \text { ) } \\
\text { Uma associação estatisticamente } \\
\text { significativa entre ansiedade } \\
\text { odontológica e cárie dentária foi } \\
\text { detectada e os escores CPO-D / ceo-d } \\
\text { foram maiores entre os pacientes } \\
\text { com níveis elevados de ansiedade } \\
\text { odontológica que entre os pacientes } \\
\text { com baixos níveis de ansiedade } \\
\text { odontológica. }\end{array}$ \\
\hline $\begin{array}{l}\text { Pezzini } \\
\text { Soares et al. } \\
\text { (13) }\end{array}$ & 2019 & Brasil & \begin{tabular}{|c} 
Ensaio \\
clínico \\
controlado \\
não \\
randomizado
\end{tabular} & 99 & 6 a 9 & \begin{tabular}{|l} 
modified Venham \\
Picture Test \\
(VPTm) \\
Respondente: \\
Criança
\end{tabular} & $\begin{array}{l}\text { Prevalência: } 40,4 \% \\
\text { A ansiedade odontológica foi } 1,99 \\
\text { vezes mais prevalente entre as } \\
\text { crianças com, ao menos, um dente } \\
\text { permanente cariado (RP = } 1,99 ; \text { IC } \\
95 \% \text { : } 1,22 \text { a } 3,23 \text { ). }\end{array}$ \\
\hline $\begin{array}{l}\text { Dahlander et } \\
\text { al. (36) }\end{array}$ & 2019 & Suécia & Coorte & 160 & 7 a 9 & $\begin{array}{c}\text { CFSS-DS } \\
\text { Respondente: Pais }\end{array}$ & $\begin{array}{l}\text { Prevalência: } 7 \% \text { das crianças aos } 7 \\
\text { anos e, aos } 9 \text { anos de idade, } 8 \% \text {. } \\
\text { Crianças que desenvolveram cárie } \\
\text { dentária entre as idades de sete e } \\
\text { nove anos tiveram } 4 \text { vezes mais } \\
\text { chance de ter medo odontológico (IC } \\
95 \% \text { : } 1,04-13,18 \text { ). }\end{array}$ \\
\hline
\end{tabular}




\section{Discussão}

Os estudos envolvendo a avaliação do medo odontológico tornaram-se mais relevantes, à medida que suas várias consequências passaram a ser melhor compreendidas pela comunidade científica (37). Pesquisas prévias confirmaram que crianças com medo odontológico apresentaram pior condição de saúde bucal $(18,28)$, posto que uma saúde bucal precária aumenta a chance de dor dentária, ou pode resultar em uma experiência odontológica negativa (38), o que pode reforçar a ocorrência do medo odontológico (28). É importante, portanto, que sejam identificados os fatores associados ao medo odontológico, para reduzir as consequências geradas pela condição, adotando-se, para isso, uma abordagem adequada.

Dos estudos selecionados nas bases bibliográficas internacionais, a maioria foi desenvolvido por pesquisadores no Brasil. Esse maior número de publicações pode estar relacionado ao progressivo interesse pela temática, associado ao crescimento da produção científica em periódicos estrangeiros, pois segundo dados do Scimago Journal \& Country Rank, o país ocupa a $2^{\circ}$ posição de publicações na área de Odontologia (39). Além disso, de acordo com o Global Burden of Disease, a ocorrência de cárie dentária no Brasil acometeu 19 milhões de crianças e adolescentes de 1 a 14 anos (40).

As pesquisas transversais se configuraram no desenho de estudo mais utilizado $(1,8,9,12,14,16-18,20-23,25-27,29,30,32-35)$. Esse tipo de estudo possibilita determinar o perfil da doença ou condição, fornece informações que determinarão o planejamento de serviços de saúde e são úteis para gerar hipóteses que podem ser confirmadas por meio de desenhos de estudo mais rigorosos, além de serem menos onerosos e mais rápidos (41). No entanto, em estudos transversais é difícil afirmar o que é a causa e o efeito, problema conhecido como causalidade reversa (41), neste caso, não sendo possível determinar se a pior condição de saúde bucal é decorrente do medo odontológico ou se o medo é resultante de experiências odontológicas negativas durante tratamentos mais invasivos devido às condições bucais mais avançadas. Deve-se estimular a realização de pesquisas que podem inferir sobre a relação de causa-efeito entre o medo odontológico e a cárie dentária, pois a identificação de fatores de risco pode ajudar na definição de estratégias mais apropriadas.

O medo odontológico pode ser mensurado através da utilização de vários métodos e, nesse estudo, o questionário mais utilizado foi o Children's Fear Survey ScheduleDental Subscale $(1,8,9,18,21,22,33-36)$, com os respondentes sendo as próprias crianças. O CFSS-DS é um instrumento largamente utilizado, para medir o medo odontológico em crianças (10), tendo sido validado em países como Holanda (42), Finlândia (43), Japão (44), Grécia (45), China (46), Índia (47) e Brasil (6), entre outros. Pode ser aplicado às crianças ou aos pais, porém a aplicação da versão parental do questionário demonstrou que pais menos ansiosos tendem a subestimar o medo dental dos filhos enquanto pais mais ansiosos tendem a superestimá-lo $(10,48)$.

A prevalência de medo odontológico e os valores de escore médio variaram amplamente em diferentes países. Essa variação reflete as diferenças metodológicas das pesquisas e entre as populações (8), referentes aos diversos instrumentos de pesquisa utilizados $(4,33)$, aos tipos de amostragem $(3,4)$ e aos pontos de corte estabelecidos $(3)$, para definir o medo odontológico, aos aspectos culturais $(3,4,8)$ e às faixas etárias avaliadas (42).

Nesta revisão, observou-se que crianças com maior experiência de cárie dentária possuem níveis mais elevados de medo (1,12-14,16-18,20-24,26,28,30,31,34-36). Entretanto, essa associação ainda é controversa na literatura, visto que alguns estudos não encontraram tal relação $(8,15,19,25,27,29,33)$.

As diferenças entre os resultados encontrados na literatura podem ser justificadas pelo 
fato de que cada população possui suas particularidades culturais $(4,8,28)$, psicossociais (3) e socioeconômicas $(28,29)$ que influenciarão na relação entre medo odontológico e cárie dentária. A diversidade no delineamento metodológico dos estudos também pode explicar essas diferenças (10).

No estudo desenvolvido por Alsadat et al. (9), o medo odontológico esteve associado com a severidade da cárie dentária; todavia, os achados de Abanto et al. (19) não demonstraram essa associação. Entre crianças indianas, a ansiedade infantil mostrou uma associação significativa com a perda dentária, sendo verificado que crianças com ansiedade odontológica têm maior probabilidade de serem submetidas a procedimentos mais traumáticos, como exodontia (32). Como consequência, o trauma gerado durante o atendimento aumenta ainda mais a ansiedade odontológica, levando a uma maior frequência de consultas perdidas, gerando um ciclo vicioso (49). Essa mesma condição também explica o elevado número de dentes perdidos entre as crianças com maior ansiedade (32). Foi sugerido ainda que a relação entre o medo odontológico e a severidade da cárie dentária pode estar associada com o fato de as crianças ansiosas comparecerem menos às consultas odontológicas (9).

Para Abanto et al. (19), outros fatores podem explicar melhor a inexistência de associação, como a idade das crianças, de modo que as crianças mais velhas experimentam menos ansiedade odontológica quando comparada às mais jovens (19) devido ao desenvolvimento de habilidades cognitivas (50). Além disso, crianças maiores compreendem melhor as explicações dadas por seus pais e cirurgiões-dentistas, reduzindo assim sua ansiedade odontológica (19).

Em estudos brasileiros, foram observadas associações significativas entre ansiedade odontológica e dentes cariados (29) e as crianças com dentes restaurados mostraram-se significativamente menos ansiosas que aquelas sem tratamento odontológico anterior (23), condição esta também observada por autores gregos os quais verificaram correlação negativa entre o medo e o número de dentes decíduos restaurados (8). Alguns estudos demonstraram que crianças que nunca foram ao cirurgião-dentista revelaram-se muito mais ansiosas quando comparadas àquelas que tinham experiência prévia $(23,51,52)$. No entanto, crianças com dentes restaurados e menos medo odontológico podem demonstrar familiaridade ao tratamento odontológico, visto que realizar o procedimento restaurador representa uma exposição e ajuda a reduzir, ou a prevenir o medo odontológico (8). Logo, a assistência odontológica, em um contexto adequado, parece ter efeitos positivos sobre o medo (23).

Portanto, os cirurgiões-dentistas devem incentivar os pais ou responsáveis a estabelecer um padrão de visita ao consultório odontológico não apenas para aqueles casos nos quais existe dor ou a doença cárie já está instalada, mas, principalmente, para ações educativas e preventivas em saúde bucal, familiarizando a criança com o ambiente do consultório, o profissional e sua equipe. Além disso, é importante conhecer os fatores que se associam ao medo odontológico, pois pode auxiliar o cirurgiãodentista a identificar, entre os seus pacientes, aqueles que são mais suscetíveis a desenvolver esse tipo de medo. Com isso, acreditamos que, através de uma abordagem apropriada, uma relação de confiança pode ser construída, melhorando as condições de saúde bucal e reduzindo a necessidade de tratamento em crianças.

\section{Conclusão}

Os estudos sobre o medo odontológico associado à experiência de cárie dentária em crianças foram realizados em maior número no Brasil, utilizando o questionário CFSS-DS. Existe ampla variabilidade na prevalência e valores de escore médio em diferentes países. 


\section{Referências}

1. Kakkar M, Wahi A, Thakkar R, Vohra I, Shukla AK. Prevalence of dental anxiety in 10-14 years old children and its implications. J Dent Anesth Pain Med 2016; 16(3): 199-202.

2. Cianetti S, Lombardo G, Lupatelli E, Pagano S, Abraha I, Montedori A, Caruso S, Gatto R, De Giorgio S, Salvato R. Dental fear/anxiety among children and adolescents. A systematic review. Eur J Paediatr Dent 2017; 18(2): 121-130.

3. Ten Berge M, Veerkamp JS, Hoogstraten J. The etiology of childhood dental fear: the role of dental and conditioning experiences. J Anxiety Disord 2002; 16(3): 321-329.

4. Klingberg G, Broberg AG. Dental fear/anxiety and dental behaviour management problems in children and adolescents: a review of prevalence and concomitant psychological factors. Int $\mathbf{J}$ Paediatr Dent 2007; 17(6): 391-406.

5. Themessl-Huber M, Freeman R, Humphris G, MacGillivray S, Terzi N. Empirical evidence of the relationship between parental and child dental fear: a structured review and meta-analysis. Int $\mathbf{J}$ Paediatr Dent 2010; 20(2): 83-101.

6. Barbério GS. Confiabilidade e validade do questionário Children's Fear Survey Schedule-Dental Subscale para avaliação do medo e ansiedade ao tratamento odontológico em crianças. Tese (Doutorado) - Faculdade de Odontologia de Bauru, Universidade de São Paulo, Bauru. 2017.

7. Armfield JM, Stewart JF, Spencer JÁ. The vicious cycle of dental fear: exploring the interplay between oral health, service utilization and dental fear. BMC Oral Health 2007; 7:1.

8. Boka V, Arapostathis K, Karagiannis V, Kotsanos N, van Loveren C, Veerkamp J. Dental fear and caries in 6-12 year old children in Greece. Determination of dental fear cut-off points. Eur J Paediatr Dent 2017; 18(1): 45-50.

9. Alsadat FA, El-Housseiny AA, Alamoudi NM, Elderwi DA, Ainosa AM, Dardeer FM. Dental fear in primary school children and its relation to dental caries. Niger J Clin Pract 2018; 21(11): 1454-1460.

10. Laureano ICCL. Correlação entre Medo Odontológico, Cárie Dentária e Hipomineralização MolarIncisivo em Escolares de 8 a 10 Anos de Idade. Dissertação (Mestrado) - Universidade Estadual da Paraíba, Campina Grande. 2019.

11. Schuch HS, Costa FS, Torriani DD, Demarco FF, Goettems ML. Oral health-related quality of life of schoolchildren: impact of clinical and psychosocial variables. Int J Paediatr Dent 2015; 25(5): 358-365.

12. Silveira ERD, Goettems ML, Demarco FF, Azevedo MS. Clinical and Individual Variables in Children's Dental Fear: A School-Based Investigation. Braz Dent J 2017; 28(3): 398-404.

13. Pezzini Soares J, Cardoso M, Bolan M. Demystifying behaviour and dental anxiety in schoolchildren during endodontic treatment for primary teeth-controlled clinical trial. Int J Paediatr Dent 2019; 29(3): 249-256.

14. Pichot H, Hennequin M, Rouchon B, Pereira B, Tubert-Jeannin S. Dental status of new caledonian children: is there a need for a new oral health promotion programme?. PLoS One 2014; 9(11): e112452.

15. Costa VPP, Correa MB, Goettems ML, Pinheiro RT, Demarco FF. Maternal depression and anxiety associated with dental fear in children: a cohort of adolescent mothers in Southern Brazil. Braz Oral Res 2017; 31: e 85.

16. Viswanath D, Krishna AV. Correlation between dental anxiety, Sense of Coherence (SOC) and dental caries in school children from Bangalore North: a cross-sectional study. J Indian Soc Pedod Prev Dent 2015; 33(1): 15-18.

17. Carrillo-Diaz M, Crego A, Armfield JM, Romero M. Dental fear-related cognitive vulnerability perceptions, dental prevention beliefs, dental visiting, and caries: a cross-sectional study in Madrid (Spain). Community Dent Oral Epidemiol 2015; 43(4): 375-384.

18. Yahyaoglu O, Baygin O, Yahyaoglu G, Tuzuner T. Effect of dentists' appearance related with dental fear and caries status in 6-12 years old children. J Clin Pediatr Dent 2018; 42(4): 262-268.

19. Abanto J, Vidigal EA, Carvalho TS, Sá SN, Bönecker M. Factors for determining dental anxiety in preschool children with severe dental caries. Braz Oral Res 2017; 31: e13.

20. Bhalla A, Rajasekaran UB, Singh M, Goutam M, Grover N, Galav A. A Cross-sectional Study to assess the Perception of Psychosocial Elements among Pediatric Patients visiting Dental Clinics. J Contemp Dent Pract 2017; 18(11):1021-1024. 
21. Beena JP. Dental subscale of children's fear survey schedule and dental caries prevalence. Eur J Dent 2013; 7(2): 181-185.

22. Akbay Oba A, Dülgergil CT, Sönmez IS. Prevalence of dental anxiety in 7- to 11-year-old children and its relationship to dental caries. Med Princ Pract 2009; 18(6): 453-457.

23. Nicolas E, Bessadet M, Collado V, Carrasco P, Rogerleroi V, Hennequin M. Factors affecting dental fear in French children aged 5-12 years. Int J Paediatr Dent 2010; 20(5): 366-373.

24. Pieper K, Weber K, Margraf-Stiksrud J, Stein S, Heinzel-Gutenbrunner M, Jablonski-Momeni, A. Evaluation of an intensified preventive programme aimed at 12 -year-olds with increased caries risk. J Public Health 2012; 20: 151-157.

25. Barbosa TS, Castelo PM, Leme MS, Gavião MB. Associations between oral health-related quality of life and emotional statuses in children and preadolescents. Oral Dis 2012; 18(7): 639-647.

26. Olak J, Saag M, Honkala S, Nõmmela R, Runnel R, Honkala E, Karjalainen S. Children's dental fear in relation to dental health and parental dental fear. Stomatologija 2013; 15(1): 26-31.

27. Murthy AK, Pramila M, Ranganath S. Prevalence of clinical consequences of untreated dental caries and its relation to dental fear among 12-15-year-old schoolchildren in Bangalore city, India. Eur Arch Paediatr Dent 2014; 15(1): 45-49.

28. Torriani DD, Ferro RL, Bonow ML, Santos IS, Matijasevich A, Barros AJ, et al. Dental caries is associated with dental fear in childhood: findings from a birth cohort study. Caries Res 2014; 48: 263-270.

29. Barreto KA, Dos Prazeres LD, Lima DS, Soares FC, Redivivo RM, da Franca C, Colares V. Factors associated with dental anxiety in Brazilian children during the first transitional period of the mixed dentition. Eur Arch Paediatr Dent 2017;18(1): 39-43.

30. Vilas-Boas AM, Vieira JOS, Diniz MB. Child's Behavior and its Relationship with the Level of Maternal-Child Anxiety During Dental Care. Pesquisa Brasileira em Odontopediatria e Clinica Integrada 2017, 17(1): e3646

31. Soares FC, Lima RA, de Barros MVG, Dahllöf G, Colares V. Development of dental anxiety in schoolchildren: A 2-year prospective study. Community Dent Oral Epidemiol 2017; 45(3): 281-288.

32. Balasubramanian S, Shrikrishna SB, Shenoy R, Rao A. Association of Maternal and Child Dental Anxiety With Dental Caries Experience and Dental Attendance Pattern of the Child. J Orofac Sci 2018; 10(2):75-79.

33. Alshoraim MA, El-Housseiny AA, Farsi NM, Felemban OM, Alamoudi NA, Alandejani AA. Effects of child characteristics and dental history on dental fear: Cross-sectional study. BMC Oral Health 2018; 18:33.

34. Folayan MO, Kolawole KA, Onyejaka NK, Agbaje HO, Chukwumah NM, Oyedele TA. General anxiety, dental anxiety, digit sucking, caries and oral hygiene status of children resident in a semiurban population in Nigeria. BMC Oral Health 2018; 18(1): 66.

35. Buldur B, Armfield JM. Development of the Turkish version of the Index of Dental Anxiety and Fear (IDAF-4C+): Dental anxiety and concomitant factors in pediatric dental patients. J Clin Pediatr Dent 2018; 42(4): 279-286.

36. Dahlander A, Soares F, Grindefjord M, Dahllöf G. Factors Associated with Dental Fear and Anxiety in Children Aged 7 to 9 Years. Dent J (Basel) 2019; 7(3): 68.

37. Armfield JM, Slade GD, Spencer AJ. Dental fear and adult oral health in Australia. Community Dent Oral Epidemiol 2009; 37(3): 220-230.

38. Boeira GF, Correa MB, Peres KG, Peres MA, Santos IS, Matijasevich A, Barros AJ, Demarco FF. Caries is the main cause for dental pain in childhood: findings from a birth cohort. Caries Res 2012; 46(5): 488-495.

39. Scimago Journal \& Country Rank. [Internet]. 2019. [citado em 2020 Out 22]. Disponível em: http://www.scimagojr.com

40. Global Health Data Exchange [Internet]. Global Burden of Disease Study 2017 (GBD 2017) Results. Seattle, WA: Institute for Health Metrics and Evaluation; 2018. [citado em 2020 Out 22]. Disponível em: http://ghdx.healthdata.org/gbd-results-tool.

41. Pandis N. Cross-sectional studies. Am J Orthod Dentofacial Orthop 2014; 146(1): 127-129.

42. Ten Berge M, Veerkamp JS, Hoogstraten J, Prins PJ. Childhood dental fear in the Netherlands: 
prevalence and normative data. Community Dent Oral Epidemiol 2002; 30(2): 101-107.

43. Alvesalo I, Murtomaa H, Milgrom P, Honkanen A, Karjalainen M, Tay KM. The Dental Fear Survey Schedule: a study with Finnish children. Int J Paediatr Dent 1993; 3(4): 193-198.

44. Nakai Y, Hirakawa T, Milgrom P, Coolidge T, Heima M, Mori Y, Ishihara C, Yakushiji N, Yoshida T, Shimono T. The Children's Fear Survey Schedule-Dental Subscale in Japan. Community Dent Oral Epidemiol 2005; 33(3): 196-204.

45. Arapostathis KN, Coolidge T, Emmanouil D, Kotsanos N. Reliability and validity of the Greek version of the Children's Fear Survey Schedule-Dental Subscale. Int J Paediatr Dent 2008; 18(5): 374-379.

46. Ma L, Wang M, Jing Q, Zhao J, Wan K, Xu Q. Reliability and validity of the Chinese version of the Children's Fear Survey Schedule-Dental Subscale. Int J Paediatr Dent 2015; 25(2): 110-116.

47. Singh P, Pandey RK, Nagar A, Dutt K. Reliability and factor analysis of children's fear survey schedule-dental subscale in Indian subjects. J Indian Soc Pedod Prev Dent 2010; 28(3): 151-155.

48. Menoncin BLV, Portella PD, Ramos BLM, Assunção LRDS, de Souza JF, Menezes JVNB. Dental anxiety in schoolchildren with molar incisor hypomineralization-A population-based cross-sectional study. Int J Paediatr Dent 2019; 29(5): 615-623.

49. Milsom KM, Tickle M, Humphris GM, Blinkhorn AS. The relationship between anxiety and dental treatment experience in 5-year-old children. Br Dent J 2003; 194(9): 503-506.

50. Laki K, Beslot-Neveu A, Wolikow M, Davit-Béal T. Child dental care: what's about parental presence? Arch Pediatr 2010; 17(11): 1617-1624.

51. Gustafsson A, Arnrup K, Broberg AG, Bodin L, Berggren U. Child dental fear as measured with the Dental Subscale of the Children's Fear Survey Schedule: the impact of referral status and type of informant (child versus parent). Community Dent Oral Epidemiol 2010; 38(3): 256-266.

52. Soares FC, Souto G, Lofrano M, Colares V. Anxiety related to dental care in children and adolescents in a low-income Brazilian community. Eur Arch Paediatr Dent 2015; 16(2): 149-152.

\author{
EDITORA E GRÁFICA DA FURG \\ CAMPUS CARREIROS \\ CEP 96203900 \\ editora@furg.br
}

